\title{
Parallel Multiple Electric Power Conversion System Constructed by Connecting Three Power Converters
}

\author{
Mitsuki Nakai*, Hiromi Inaba**, Keiji Kishine** and Keisuke Ishikura**
}

\begin{abstract}
The electric power conversion system constructed by connecting two or more power converters in parallel is an advantageous method for making to large capacity and standardization. In this paper, the control method of cross current when three power converters are operated is examined, and it reexamined a preferable system construction method.
\end{abstract}

Keywords: Power converter, Parallel connecting, Cross current, Combination reactor

\section{Introduction}

In a parallel operation system, the combination reactor is required for compounding the output of two or more sets of unit electric power converter. Combination reactor is added the passive suppression function of the cross current unrelated to a motor drive. However, the miniaturization of a combination reactor is important in the system which mounted in the limited space with high density.

Then, summation and difference current control which suppress the cross current by independent control of motor current (summation ingredient) and cross current (difference ingredient) is proposed for a combination reactor is miniaturized [1].

Moreover, in almost all the parallel operation system, the electric power converter which constitutes a system was examination by equal output current assignment.

Since the flexibility of a systems configuration will increase if the extension to odd sets can do combination of multiple connection, it becomes still more advantageous from a viewpoint of standardization. There is a system which combines three sets of electric power converters as a trial which extend the combination of multiple connection to odd sets [2]. The method using summation and difference current control is achieved by a parallel operation system of the double structure.

In this paper, the problem of the previous current control system was clarified from a viewpoint of cross current suppression about the parallel operation system of three sets of electric power converters. Furthermore, we propose an

* Ishida Co., Ltd., Japan. (mimimi.mitsuki@ gmail.com)

** Dept. of Electronic Systems Engineering, the University of Shiga Prefecture, Japan. (inaba.h@usp.ac.jp, kishine.k@usp.ac.jp,zn23kishikura@ec.usp.ac.jp)

Received 11 April 2014; Accepted 13 June 2014 improvement proposal and the circuit simulation estimated.

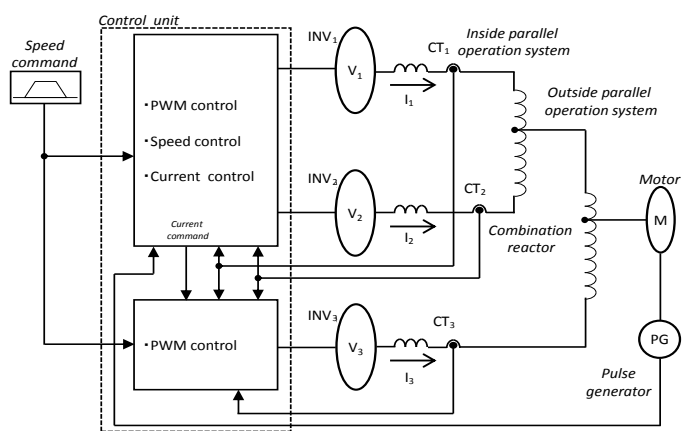

Fig. 1. Outline of the overall structure

\section{The Whole System Composition}

Fig.1 shows the overall structure of three parallel connected converters. The output of each electric power converter is compounded with a combination reactor, and is inputted into a motor. Motor speed is measured with a pulse generator, and the converter output currents I1, I2 and I3 are measured with current detectors CT1, CT2 and CT3 respectively. Speed and current information is sent to a main controller (command generator). The main controller processes speed control (ASR) and current control (ACR). PWM pulse instruction is provided to INV1, INV2 and INV3 to control the output voltages of the converters.

\section{The Problem of the Previous Current Control System}

Fig. 2 shows the previous current control system which combines three sets of electric power converters. The 
feature of the previous system is performing summation and difference current control separately on the inside and the outside. That is, inside parallel operation system is one converter assumes.

This composition has two or more summation current control systems. For this reason, it is known that the inside cross current is increased by outside control system interfere the inside control system.

Therefore we aim at the reduction of the cross currents by controlling inside and outside current controls making to the non-interactive control.

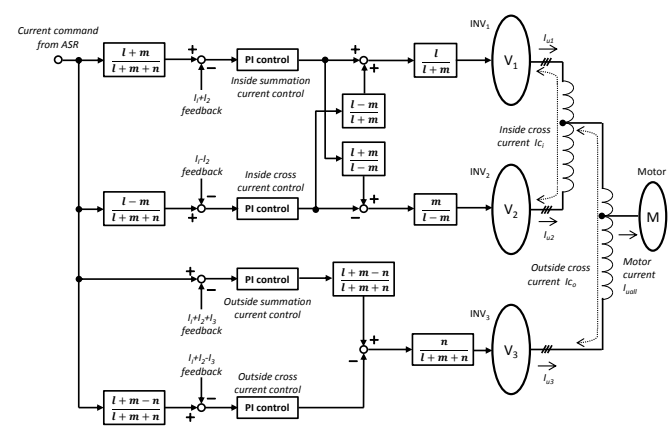

Fig. 2. Block diagram of previous current control system

\section{Proposal Current Control System}

Fig.3 shows the proposal current control system. Each current control system was prevented from mutually interfering by uniting two or more summation current control systems that were to one in the proposal system. The cross current suppression control is done respectively by inside parallel driving and outside parallel driving conventional. But, outside control system does not affect the inside control system because cross current suppression control is decouple to the summation current control in the proposed system. Moreover, since a proposal system is simple composition compared with the previous system, the number of times of operation not only becomes fewer, but there is a merit that software can be made easily.

\section{The Valuation Method by a Simulation}

The proposal system was compared with the previous system by the circuit simulation. The main circuit unit had the same composition, built the previous system and the proposal system on a software package PSIM of Powersim Inc., and performed the simulation to a trapezoid speed instruction pattern.

Fig.4 shows the main circuit unit. The main circuit unit consists of a PM motor and an electric power converter which is the drive circuit. Although the electric power converter consists of a converter and an inverter, order conversion is imitating as what is performed ideally by the direct-current power supply this time.

Fig.5 shows the control part of a previous system. Fig.6 shows the control part of a proposal system. The previous system and a proposal system consist of ASR and ACR. It turns out that the number of times of operation of a proposal system of PI control and summation and difference current control in ACR is decreasing.

Moreover, the composition by the electric power converter of capacity which is different by setting output current assignment instructions of INV1, INV2, and INV3 to $2: 1: 1$ was imitated, and unbalance was given to all during three output current assignments.

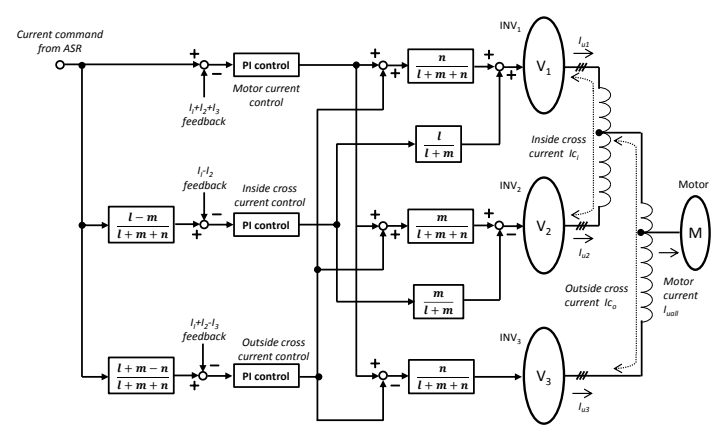

Fig. 3. Block diagram of proposal current control system

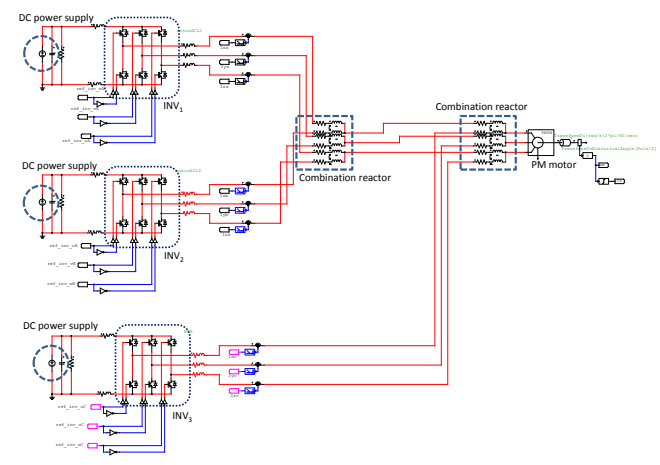

Fig. 4. Main circuit unit

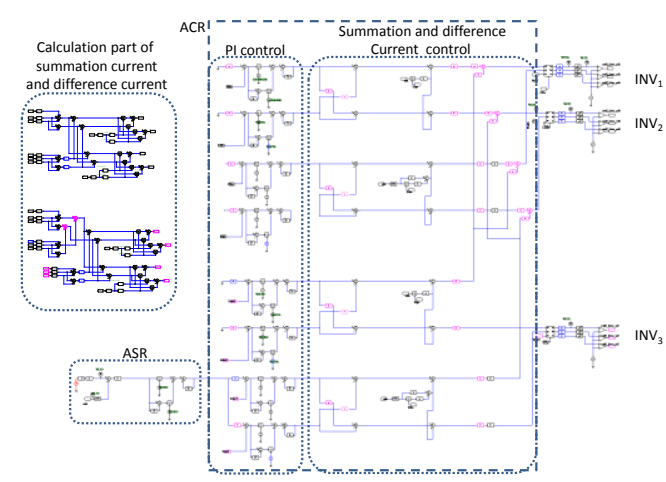

Fig. 5. Control unit of a previous system 


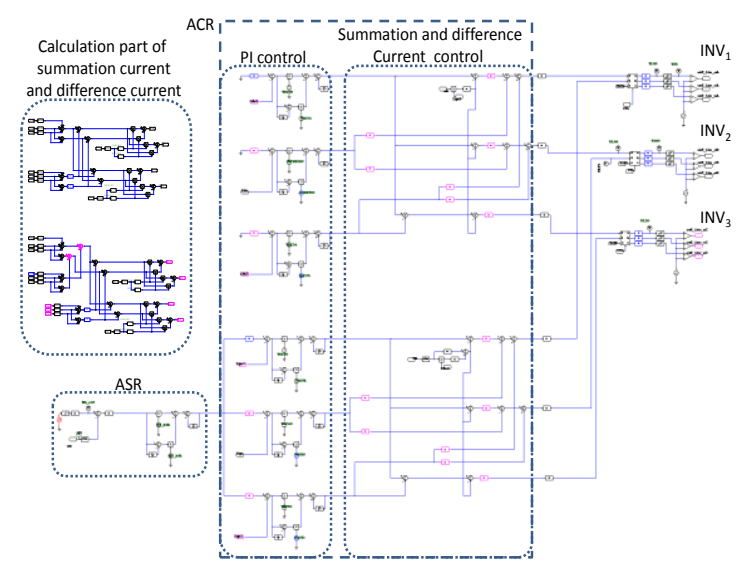

Fig. 6. Control unit of a proposal system

\section{Comparison in the Ideal State}

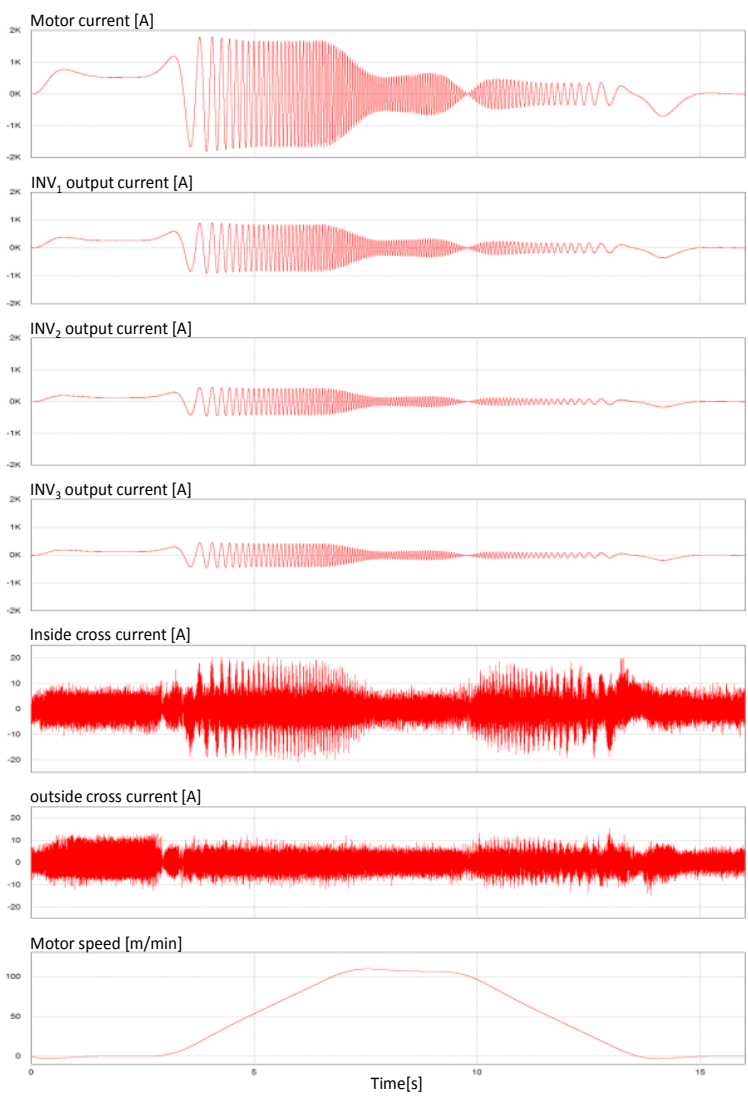

Fig. 7. Simulation result under ideal condition in the case of previous system

The simulation in the case where all of the element and power supply which constitute an electric power converter are in ideal conditions is carried out. The simulation result shows U phase among each current ingredient.

Fig.7 shows the motor current, output current of INV1, output current of INV2, output current of INV3, inside cross current, outside cross current and motor speed in a previous system.
Fig.8 shows the motor current, output current of INV1, output current of INV2, output current of INV3, inside cross current, outside cross current and motor speed in a proposal system.

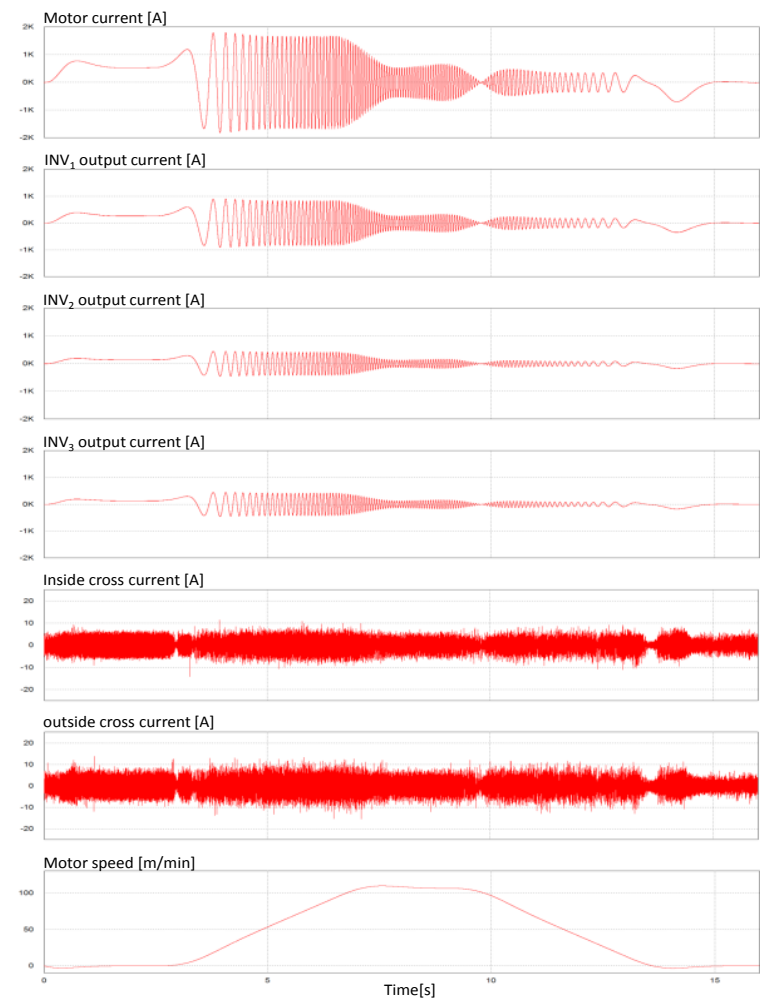

Fig. 8. Simulation result under ideal condition in the case of proposal system

First, a summation current control system is compared.

About the effective value of each summation current ingredient of the previous system, motor current is $688.29 \mathrm{~A}$ output current of INV1 is $344.24 \mathrm{~A}$, output current of INV2 is $172.04 \mathrm{~A}$, and output current of INV3 is $172.01 \mathrm{~A}$. About the effective value of each summation current ingredient of the proposal system, motor current is 688.29A, output current of INV1 is $344.24 \mathrm{~A}$, output current of INV2 is $172.03 \mathrm{~A}$, and output current of INV3 is $172.001 \mathrm{~A}$.

As for the summation current control, both of the systems had realized 2:1:1 of the output current assignment ratio, and the difference was not looked at by the waveform.

Next, a cross current suppression control system is compared. In the case that the inside cross current of Fig.7 and Fig. 8 is compared, the difference appears notably in the domain in which speed changes. Is suppressed the maximum value of the cross current proposed system than the previous system.

About the effective value of each cross current ingredient of the previous system, inside current is $3.55 \mathrm{~A}$, outside cross current is $3.07 \mathrm{~A}$, and the sum total is $6.6 \mathrm{~A}$. About the effective value of each cross current ingredient of the 
proposal system, inside current is $2.56 \mathrm{~A}$, outside cross current is $3.18 \mathrm{~A}$, and the sum total is $5.74 \mathrm{~A}$.

Even if it compares the sum total value of a cross current from a viewpoint of efficiency, it turns out that the cross current of a proposal system has decreased compared with the previous system. Although the difference between two systems with a suppression effect of an outside cross current is not seen, it can be said that the proposal system of the suppression effect of an inside cross current is higher.

From the above thing, the proposed system can be confirmed that the cross current suppression function is improved than the previous system by the non-interactive current control system without doing not impair the controllability of the motor.

\section{Influence on the Cross Current Inhibitory Control System by the Power Supply Error of an Inverter}

Potential difference occurs between the DC power supplies to each converter in an actual parallel connected power converters because of what changed AC voltage into DC voltage with the converter is used for DC power supply of an converter. It has possibility that potential difference in the output of the converter in each will affect the cross current suppression.

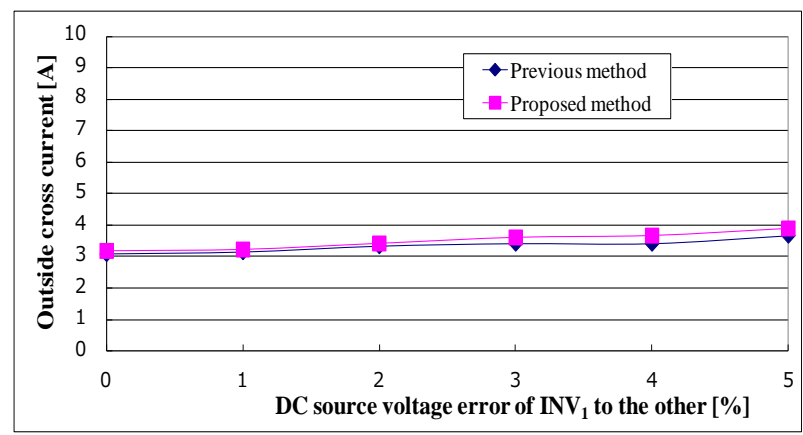

Fig. 9. Change in inside cross current in the case of the power supply error of INV1 is given

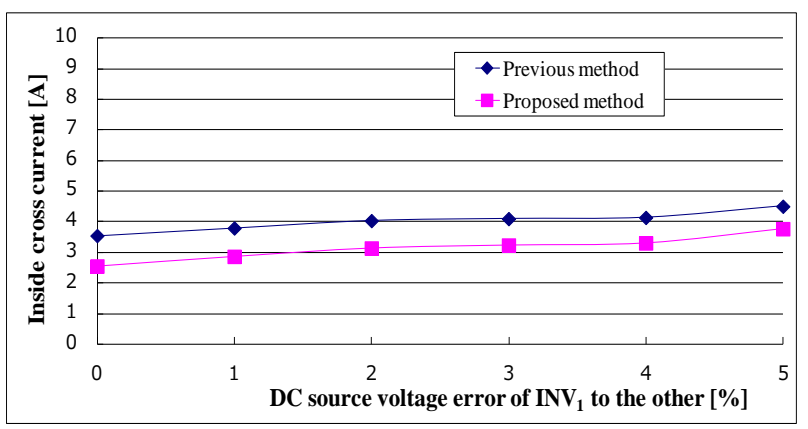

Fig. 10. Change in outside cross current in the case of the power supply error of $\mathrm{INV}_{1}$ is given
In this chapter, two control systems are compared about the influence which error of DC power supply has on the cross current suppression control. In the simulation, it imitated by giving an intentional error to the DC power supply supplied to INV1 and INV3 in Fig.4.

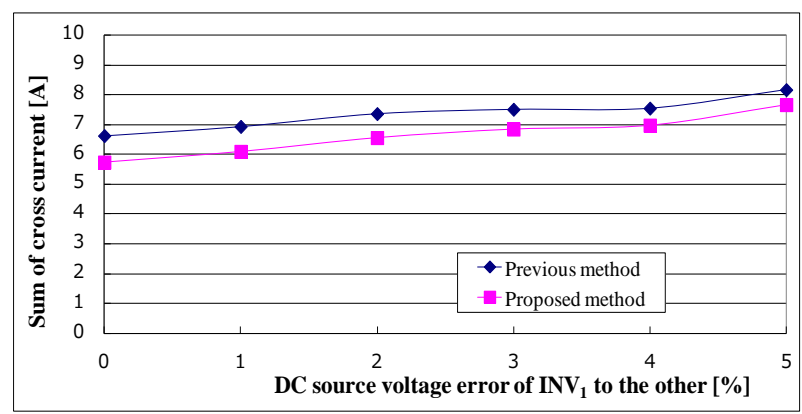

Fig. 11. The total sum of cross current in the case of the power supply error of INV1 is given

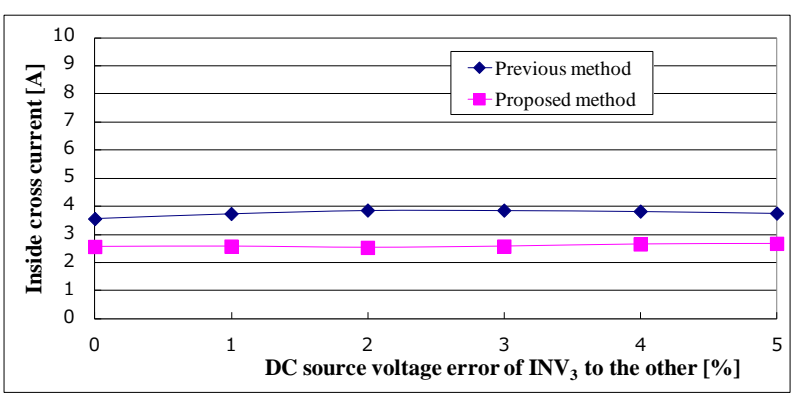

Fig. 12. Change in inside cross current in the case of the power supply error of $\mathrm{INV}_{3}$ is given

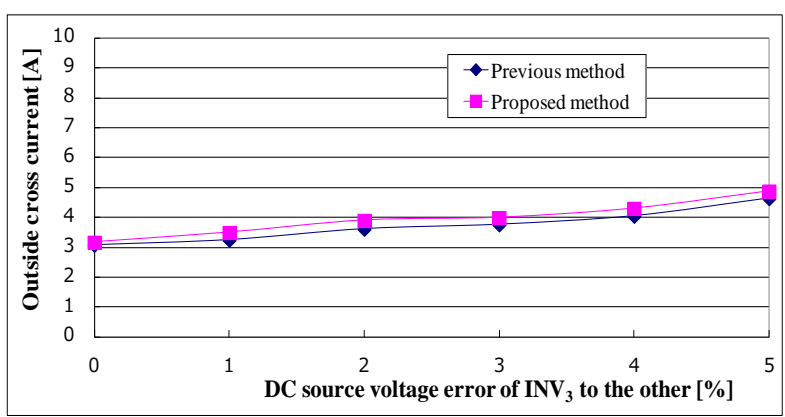

Fig. 13. Change in outside cross current in the case of the power supply error of $\mathrm{INV}_{3}$ is given

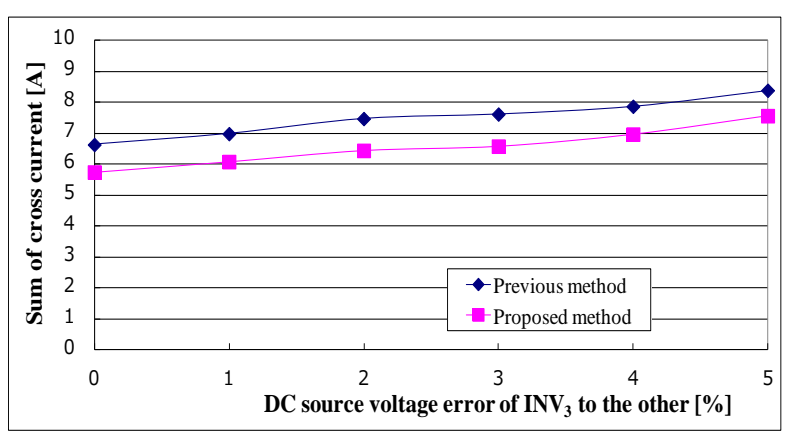

Fig. 14. The total sum of cross current in the case of the power supply error of $\mathrm{INV}_{3}$ is given 
In the case of the power supply error of INV1 is given, change in the inside cross current is shown in Fig.9, change in the outside cross current is shown in Fig.10, and the sum total of the cross current is shown in Fig. 11.

In Fig.9 and Fig.10, compared with the previous system as for proposal system, it turns out that the suppression effect of the inside cross current is high and the suppression effect of the outside cross current is slightly low. But, the cross current suppression effect as the whole system can be said to be that the direction of a proposal system is high.

In the case of the power supply error of INV3 is given, change in the inside cross current is shown in Fig.12, change in the outside cross current is shown in Fig.13, and the sum total of the cross current is shown in Fig.14.

In the case that the cross current suppression effect of the previous system and the proposal system was compared, it was the same result as the case where an error is given to the power supply of INV1. In Fig.12 and Fig.13, since the outside control system interferes inside control system, the power supply error of INV3 increases, and an inside cross current increases in the previous system. Inside cross current control is not affected because the outside control system doesn't interfere inside control system.

\section{Conclusion}

The cross current suppression control method of the parallel, multiple electric conversion system constructed with three converters was examined. It was clarified that the proposal method is less operation number of times than the previous method and increases the suppression performance of cross current.

\section{Acknowledgements}

The authors would like to thank H. Ayano of Tokyo National College of Technology and T. Yoshikawa of Hitachi, Ltd. for their helpful discussions and suggestions. A part of this work was supported by Grant-in-aid for Scientific Research(C).

\section{References}

[1] H. Inaba et al, "An Electric Power Conversion System Composed of Two Parallel-Connected Converters Providing Different Output Currents, " ICEMS, DS4G4-2, 2012.

[2] M. Nakai, et. al., "Parallel Connected Power Converter Constructed with Odd Number Converters," in Japan Ind. Appl. Society Conf. Rec., 2012.
[3] H. Anayo, et. al., "Electrical Driving Techniques for LargeScale and Ultra-High Speed Elevators," Journal of the AEM(Japan), vol.16, no.1, 2008, pp.1-6.

[4] H. Inaba, et. al., "A Study on Construction and Control Method of Parallel Connected Power Converter," in IEEJ Annual. Meeting Rec., 4-069, 2011, p128.

[5] H. Inaba, et. al., "Simulation Analysis of Construction and Control Method of Parallel Connected Power Converter," in Japan Ind. Appl. Conf. Rec., 1-2, 2011, pp.I-103-106.

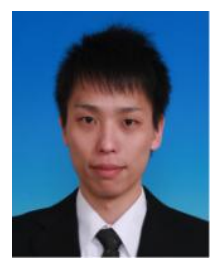

Mitsuki Nakai received the B.S. and M.S. degrees of electronic systems engineering from The University of Shiga Prefecture in 2012 and 2014. In 2014, he joined Ishida Co., Ltd., Shiga, Japan. His research interest is a parallel connected power converter and motor drive.

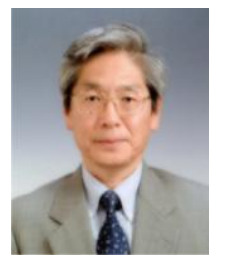

Hiromi Inaba was born in Tokyo, Japan, on September 1, 1950. He received the B.S and Dr. Eng. degrees from Hokkaido University, Hokkaido, Japan, in 1974 and 1997, respectively. In 1974 he joined Hitachi Research Laboratory, Hitachi, Ltd., Ibaraki, Japan. He has been engaged in research and development on an elevator and steel control system. In 2008, he became a Professor with the school of engineering, The University of Shiga prefecture, Shiga, Japan. Dr. Inaba is a member of the IEEE Industry Applications Society (IAS) and the Institute of Electrical Engineers (IEE) of Japan.

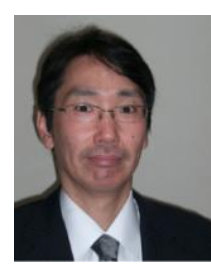

Keiji Kishine was born in Kyoto, Japan, on October 26, 1964. He received the B.S., and M.S. degrees in engineering science from Kyoto University, Kyoto, Japan, and $\mathrm{Ph} . \mathrm{D}$. degree from in informatics from Kyoto University, Kyoto, Japan, in 1990, 1992, and 2006, respectively. In 1992, he joined the Electrical Communication Laboratories, Nippon Telegraph and Telephone Corporation (NTT), Tokyo, Japan. He has been engaged in research and design of high-speed, lowpower circuits for Gbit/s LSIs using Si-bipolar transistors, with application to optical communication systems in NTT 
System Electronics Laboratories, Kanagawa, Japan. From 1997, he has been worked on research and development of over Gbit/s Clock and Data Recovery IC at Network Service Innovation Laboratory in NTT Network Innovation Laboratories, Kanagawa, Japan. Now, He is working at Ubiquitous Interface Laboratory in NTT Microsystems Integration Laboratories, Kanagawa, Japan. In 2008, he became an Associate Professor with the school of engineering, The University of Shiga prefecture, Shiga, Japan. Dr. Kishine is a member of the IEEE Solid-State Circuits Society (SSCS) and Circuits and Systems (CAS), the Institute of Electronics, Information and Communication Engineers (IEICE) of Japan, the Institute of Electrical Engineers (IEE) of Japan.

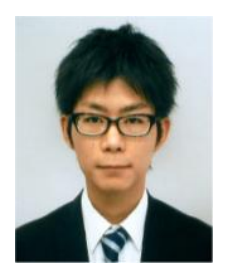

Keisuke Ishikura received the B.S. degree of electronic systems engineering from The University of Shiga Prefecture in 2013. Since the same year, he has enrolled a master's course Graduate school of Engineering in The University of Shiga Prefecture. His research interest is a parallel connected power converter and motor drive. 